\title{
Senecio brasiliensis e alcaloides pirrolizidínicos: toxicidade em animais e na saúde humana
}

\author{
Thaisa Meira Sandini ${ }^{1 *}$ \\ Mariana Sayuri Udo Berto ${ }^{1}$ \\ Helenice de Souza Spinosa ${ }^{2}$ \\ Universidade de São Paulo \\ ${ }^{1}$ PPG em Toxicologia e Análises Toxicológicas, Faculdade de Ciências Farmacêuticas \\ Avenida Professor Lineu Prestes, 580, CEP 05508-000, São Paulo - SP, Brasil \\ ${ }^{2}$ Departamento de Patologia, Faculdade de Medicina Veterinária e Zootecnia \\ * Autor para correspondência \\ thaisasandini@usp.br
}

Submetido em $17 / 08 / 2012$

Aceito para publicação em 08/02/2013

\section{Resumo}

No Brasil, as plantas tóxicas são responsáveis por grande parte dos prejuízos econômicos em animais de produção, principalmente em equinos e bovinos. Nesse contexto, no Rio Grande do Sul, o gênero Senecio, especialmente $S$. brasiliensis Lessing, merece maior destaque por ser a principal causa de mortes de bovinos adultos. A toxicidade causada por esse gênero vegetal ocorre devido à presença dos alcaloides pirrolizidínicos, os quais sofrem biotransformação no fígado, gerando metabólitos tóxicos: os pirrois. Esses compostos tóxicos podem também ser nocivos para o homem através do consumo de produtos comestíveis, de origem animal, contaminados ou pelo uso dessa planta na medicina popular. Assim, o presente artigo traz uma revisão, em particular de $S$. brasiliensis com ênfase nos seus princípios ativos tóxicos e no mecanismo pelo qual se desenvolve a patogenia, além de abordar outras plantas que contêm os mesmos princípios tóxicos e que causam efeitos nocivos à saúde humana.

Palavras-chave: Alcaloides pirrolizidínicos; Animais de produção; Humanos; Senecio brasiliensis; Toxicidade

\section{Abstract}

Senecio brasiliensis and pyrrolizidine alkaloids: toxicity to animals and human health. Most economic losses in Brazilian livestock production, especially in horses and cattle, are due to poison plants, such as those of the genus Senecio. Senecio brasiliensis Lessing is the main cause of death in cattle in the state of Rio Grande do Sul. The toxicity of this genus is attributed to their content of pyrrolizidine alkaloids, which undergo liver biotransformation creating toxic metabolites, namely pyrroles. These compounds can be transferred to humans through contaminated animal products or by the use of this plant in folk medicine. Thus, the present article is a review of the species $S$. brasiliensis, its toxic active principles and the mechanism by which pathogenesis occurs. Other plants with the same toxic principles that are harmful to human health are covered as well.

Key words: Human; Livestock; Pyrrolizidine alkaloids; Senecio brasiliensis; Toxicity 


\section{Introdução}

As intoxicações por plantas em animais de produção no Brasil são conhecidas desde a época da colonização do nosso país e, ainda nos dias de hoje, estão entre as causas mais comuns de morte de bovinos adultos, resultando em perdas econômicas diretas e indiretas. Ressalta-se que, no estado do Rio Grande do Sul a intoxicação atribuída às plantas é uma das principais causas de morte entre bovinos (RIET-CORREA; MEDEIROS, 2001; BARROS et al., 2007; LUCENA et al., 2010; KARAM; MOTTA, 2011). Estima-se que metade dessas mortes é causada por diferentes espécies de Senecio, totalizando uma perda anual de US\$ 7,5 milhões (MÉNDEZ; RIETCORREA, 2008). Especula-se que estes valores sejam ainda mais elevados, uma vez que estes dados foram baseados apenas na morte de animais.

Considerando que o gênero Senecio possui uma grande variedade de espécies, sendo a $S$. brasiliensis Lessing a principal delas associada às perdas econômicas em agropecuária no Brasil, este artigo de revisão tem como objetivo abordar a toxicidade dessa espécie, bem como de outras que contêm os mesmos princípios tóxicos e que causam efeitos nocivos à saúde humana.

Esse artigo envolveu levantamento bibliográfico, tendo como fontes bibliográficas livros de divulgação científica, periódicos, dissertações, teses e obras de referência clássica. As fontes bibliográficas foram obtidas em bibliotecas convencionais, nas bases de dados eletrônicas PubMed, SciELO, Scopus e ScienceDirect, e os sistemas de busca foram Google Acadêmico e Google, sendo utilizadas as seguintes palavras chaves: Senecio, Senecio brasiliensis, alcaloides pirrolizidínicos e pyrrolizidine alkaloids.

\section{Sobre o gênero Senecio sp., descrição e distribuição}

As espécies do gênero Senecio sp., pertencentes a família Asteraceae (Compositae), são nativas de várias regiões do mundo; são consideradas causadoras de enfermidades de caráter enzoótico em bovinos (SANTOS et al., 2008), podendo também causar toxicidade em seres humanos (STEGELMEIER, 2011).
Mundialmente são descritas mais de 1200 espécies de Senecio. Destas, sabe-se que cerca de 25 espécies são comprovadamente tóxicas para os animais domésticos e para o homem (TOKARNIA; DÖBEREINER, 2000). Motidome e Ferreira, em 1966, relataram que no Brasil havia aproximadamente 128 espécies de Senecio; depois Cabrera e Klein, em 1975, apontaram haver apenas 85. Posteriormente, a literatura relata a ocorrência de 67 espécies, em função da transferência de algumas delas para outros gêneros (HIND, 1993).

A espécie mais frequente no Brasil é o Senecio brasiliensis, conhecido popularmente como "flor das almas", "tasneirinha" e principalmente "Maria-mole" (RIET-CORREA et al., 1993; KARAM, et al., 2002; GRECCO et al., 2010). Em nosso país, S. brasiliensis apresenta distribuição geográfica principalmente na região sul, podendo ser observada também em áreas altas e frescas da região sudeste (PILATI; BARROS, 2007).

Senecio brasiliensis é uma planta perene, herbácea, ereta, de até $2 \mathrm{~m}$ de altura; possui caule glabro, cilíndrico, geralmente ramoso na parte superior; folhas alternadas, pecioladas, com a face inferior branco-pubescente e inferior glabra, de $10-20 \mathrm{~cm}$ de comprimento; capítulos radiados, com 40 - 50 flores de coloração amarela odoríferas; o fruto é um aquênio pequeno, cilíndrico, cinza, ou pardo escuro (CORRÊA, 1984; LORENZI, 1994; SOUZA, 2007).

Esta planta apresenta facilidade de propagação, principalmente sob condições ambientais ideais, como umidade e luz para a brotação, e temperatura para floração, favorecendo sua ingestão pelos animais em qualquer período do ano (KARAM et al., 2002). Apesar da baixa palatabilidade, o consumo desta pelos bovinos no Rio Grande do Sul ocorre principalmente entre os meses de maio a agosto, período no qual a disponibilidade de pastagem diminui e as plantas estão em brotação (BARROS et al., 1987; MÉNDEZ; RIETCORREA, 2008; SPINOSA et al., 2008).

\section{Sobre princípios ativos, mecanismo de ação e patogenia}

As plantas do gênero Senecio possuem como princípios ativos tóxicos os alcaloides pirrolizidínicos 
(APs). Esses alcaloides por si só não apresentam toxicidade, porém se tornam tóxicos quando biotransformados no fígado a uma forma pirrólica altamente reativa, conhecida como de-hidropirrolizidinas, que são os metabólitos tóxicos primários, e como secundários, o álcool pirrol (PRAKASH et al., 1999).

A biossíntese dos APs tem início nas raízes da planta, onde são inicialmente produzidos os N-óxidos da senecionina e estes são transportados para estruturas superiores, isto é, para as folhas e flores, onde sofrem alterações moleculares, originando os diferentes APs (MACEL et al., 2004).

Segundo Moraes (1952), os maiores teores de alcaloides são encontrados quando a planta está em período de floração, porém estudos realizados com sementes mostraram que essas seriam as partes mais ricas em APs, indicando que a planta madura é mais tóxica (CONNOR, 1977). Mais tarde, novas pesquisas foram realizadas nos Estados Unidos com o objetivo de analisar o teor de APs presentes em diversas espécies de Senecio; concluiu-se que o conteúdo de alcaloides em cada uma das espécies estudadas varia muito durante o ciclo de crescimento da planta e de ano para ano (JOHNSON et al., 1985). Nesses estudos, também foi verificado que as flores da maioria das espécies tóxicas de Senecio continha mais APs do que nas folhas e caules, evidenciando, dessa forma, que há uma grande variação da quantidade do princípio tóxico nas diferentes partes da planta.

Em relação a $S$. brasiliensis, esta apresenta como principais APs a integerrimina e a senecionina, e como alcaloide secundário a retrorsina (MOTIDOME; FERREIRA, 1966; TRIGO et al., 2003). Quanto à toxicidade, todas as partes de $S$. brasiliensis são tóxicas, tanto verdes quanto dessecadas (TOKARNIA; DÖBEREINER, 2000). No que se refere à variação de toxidez de acordo com a fase de desenvolvimento, alguns estudos mostraram que a $S$. brasiliensis apresenta maior concentração de APs no período de floração (MORAES, 1952; KARAM et al., 2002; 2004).

Quimicamente, os APs são moléculas heterocíclicas que contém um nitrogênio ligado ao anel principal e geralmente são substâncias de caráter alcalino. $\mathrm{O}$ núcleo básico de aminoálcool recebe o nome de necina (núcleo pirrolizidina), sendo constituído por dois aneis de cinco átomos unidos por um único átomo de nitrogênio; a porção ácida é chamada de ácido nécico (ácido alifático) e é composta por uma ou duas ramificações de éster carboxílico (Figura 1) (HENRIQUES et al., 2004; CHEN; HUO, 2010).

FIGURA 1: Estrutura dos alcaloides pirrolizidínicos.

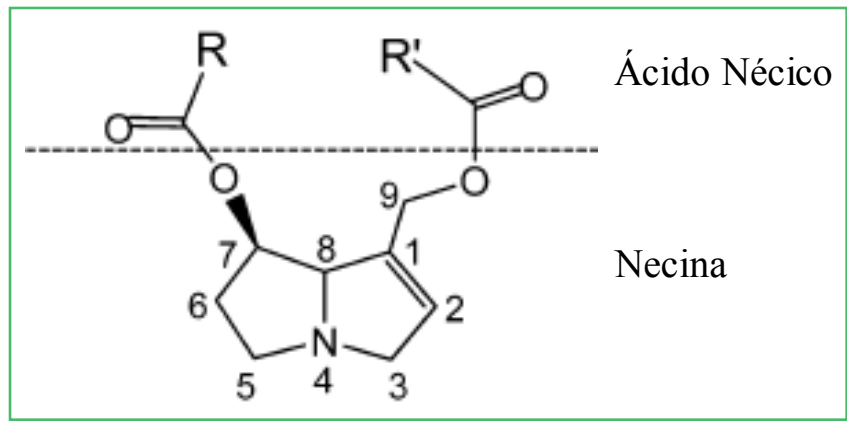

A estrutura básica responsável pela hepatotoxicidade dos APs é determinada por quatro características estruturais mínimas (PRAKASH et al., 1999): 1) um anel 3-pirrolina; 2) um ou dois grupamentos hidroxilas ligado ao anel pirrolina; 3) um ou dois grupamentos esterificados; e 4) uma cadeia ramificada no resíduo ácido (Figura 2). No entanto, a característica imprescindível para a toxicidade hepática, tanto aguda quanto crônica, é a necina insaturada entre os carbonos 1 e 2 (CHEEKE, 1988).

FIGURA 2: Características estruturais essenciais para a toxicidade dos alcaloides pirrolizidínicos: 1) anel 3-pirrolina; 2) grupamentos hidroxilas ligado ao anel pirrolina; 3 ) grupamentos esterificados; e 4) cadeia ramificada no resíduo ácido.

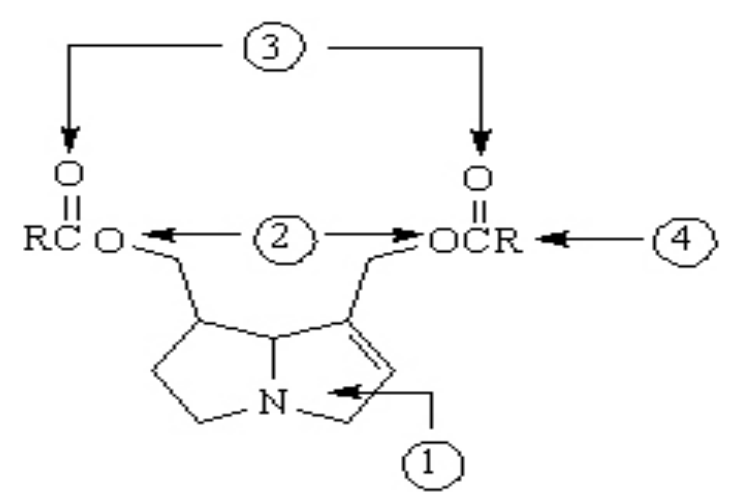


Após a absorção, a circulação portal transporta os APs até o fígado onde são biotransformados pelas enzimas microssomais hepáticas do citocromo $\mathrm{P} 450$, originando os pirrois, compostos altamente tóxicos (MATTOCKS et al., 1986). Os processos de biotransformação dos APs são realizados por três reações principais: hidrólise, oxidação e desidrogenação (MATTOCKS et al., 1986; FU et al., 2002). As duas primeiras reações estão envolvidas com o mecanismo de detoxificação, enquanto a desidrogenação está relacionada com a formação dos pirrois, os quais são responsáveis pela toxicidade das plantas do gênero Senecio (SANTOS et al., 2008).

A toxicidade dos pirrois está relacionada com sua alta reatividade, agindo como agentes alquilantes e ligando-se facilmente às moléculas de DNA. Além disso, os pirrois atuam inibindo a mitose nos hepatócitos, levando a megalocitose, necrose e, consequentemente, redução do número de hepatócitos, os quais são substituídos por tecido conjuntivo fibroso, dando início ao quadro clínico e a morte, devido à disfunção hépática (MATTOCKS, 1968; SANTOS et al., 2008). Com relação às lesões hepáticas, estas podem afetar as células parenquimatosas (hepatócitos), não parenquimatosas (células de Kupffer, células Ito, endoteliais, células de ductos biliares e linfócitos associados ao fígado), bem como a matriz extracelular hepática (SHIRATORI et al., 1993; TORRES; COELHO, 2008). Parte dos pirrois pode escapar para a circulação geral, causando lesões em outros tecidos, como, por exemplo, renal e pulmonar (RADOSTITS et al., 2002; BASILE et al., 2005; PILATI; BARROS, 2007), uma vez que as enzimas do citocromo P450 também estão presentes nestes órgãos (CULLEN, 2009).

\section{Sobre a toxicidade em animais}

Até a década de 1980 sabia-se pouco sobre a toxicidade de $S$. brasiliensis para os animais domésticos no Brasil. Os poucos estudos sobre a intoxicação desta planta, em condições naturais de exposição, se limitavam às espécies equina e bovina (TOKARNIA; DÖBEREINER, 2000). De fato, o primeiro surto da intoxicação foi relatado em 1946, em equinos alimentados com alfafa contaminada por $S$. brasiliensis, no estado de São Paulo (CARVALHO; MAUGE, 1946).
Mais tarde, no estado do Paraná, foram relatados casos de cirrose hepática em cavalos, provavelmente, por ingestão de Senecio (CURIAL; GUIMARÃES, 1958). Já em relação aos bovinos, foram relatadas suspeitas e casos diagnosticados de intoxicação por Senecio na região sul do Brasil a partir da década de 1980 (RIETCORREA et al., 1983; KARAM et al., 2004; BASILE et al., 2005; RISSI et al., 2007; GRECCO et al., 2010; LUCENA et al., 2010).

A exposição prolongada a espécies de Senecio leva a uma doença progressiva conhecida por seneciose, na qual os sinais clínicos podem ser observados várias semanas ou até meses após a ingestão da planta (RIETCORREA et al., 1998; KARAM et al., 2002). O primeiro sinal clínico observado nos animais intoxicados é a perda de peso devido à diminuição da ingestão de alimentos (RIET-CORREA et al., 1998; BASILE et al., 2005).

Em casos de intoxicação em bovinos e equinos são descritos alguns achados neurológicos, como lesões histológicas no sistema nervoso central, caracterizadas por discretos focos de malácia, leve satelitose, edema de meninge, entre outros (BARROS et al., 1987; GAVA; BARROS, 1997; BASILE et al., 2005; PILATI; BARROS, 2007). Essas lesões histológicas não são provocadas pela ação direta dos APs, mas devido à incapacidade do fígado em biotransformar a amônia. Assim, a amônia se acumula no encéfalo, reagindo com o ácido $\alpha$-cetoglutárico para formar a glutamina. A depleção do ácido $\alpha$-cetoglutárico, um intermediário do ciclo do ácido cítrico, prejudica a formação de adenosina trifosfato (ATP), reduzindo os seus níveis e assim comprometendo o metabolismo cerebral (CHEEKE, 1998). Em razão disto a hiperamonemia determina o quadro clínico denominado encefalopatia hepática.

Em equinos foram descritos casos de intoxicação espontânea e experimental por $S$. brasiliensis (GAVA; BARROS, 1997; PILATI; BARROS, 2007; TORRES; COELHO et al., 2008). Nesses animais, achados de necropsia mostraram icterícia, ascite e megalocitose de hepatócitos; observou-se, também, que alguns animais apresentaram distúrbios neurológicos, como hiperexcitabilidade, andar cambaleante e incoordenação motora (PILATI; BARROS, 2007; SANTOS et al., 2008). 
Considerando a importância econômica da intoxicação ocasionada por plantas do gênero Senecio em bovinos, estudos foram conduzidos com $S$. brasiliensis. Assim, constatou-se que a morbidade em bovinos variou entre $1 \%$ a $30 \%$ e a letalidade foi quase $100 \%$ (MÉNDEZ et al., 1990; BASILE et al., 2005). A sintomatologia observada nesses animais foi perda de peso, tenesmo e prolapso retal, pelagem grossa, distúrbios digestivos e, ocasionalmente, icterícia, fotossensibilização e edema cutâneo da barbela (BASILE et al., 2005).

Os achados anatomopatológicos descritos em bovinos foram fibrose hepática, bem como tumefação da superfície capsular, acentuação do padrão lobular, presença de nódulos regenerativos e diversos graus de degeneração, megalocitose e necrose de hepatócitos; observou-se ainda icterícia, ascite, edema e hemorragia no mesentério e nas paredes dos intestinos grosso e delgado (RIET-CORREA et al., 1998; PILATI; BARROS, 2007).

A intoxicação em bovinos ocorre principalmente em animais adultos, em particular em vacas por permanecerem por maior tempo na propriedade e ingerirem, ao longo do tempo, maior quantidade de Senecio (MÉNDEZ; RIET-CORREA, 2008). Maclachlan e Cullen (1998) relatam que os machos são menos susceptíveis do que as fêmeas e os bovinos jovens são mais sensíveis à intoxicação do que os animais adultos. Por outro lado, num estudo epidemiológico com bovinos intoxicados por Senecio spp., observou-se que dos 35 surtos avaliados $34 \%$ ocorreram em animais abaixo de três anos e 60\% em animais adultos (GRECCO et al., 2010).

Estudos recentes realizados em ratos mostraram que a exposição pré-natal ao $S$. brasiliensis prejudicou o desenvolvimento físico e reflexológico da prole (DALMOLIN et al., 2010; SANDINI et al., 2011). Esses achados sugerem que esse dano pode também ocorrer em animais de produção, principalmente bovinos, uma vez que $S$. brasiliensis é muito frequente nas pastagens, podendo ser ingerida pelos animais em condições naturais e em idade fértil. Por outro lado, Elias et al. (2011), avaliando o sistema imune de ratos adultos expostos à integerrimina $\mathrm{N}$-óxido, obtida de S. brasiliensis, não verificaram efeitos marcadamente imunotóxicos. Assim, pode-se especular que o sistema nervoso central seja mais sensível aos efeitos tóxicos desta planta quando comparado ao sistema imunológico.

Em ovinos, estudos experimentais mostraram que a intoxicação por $S$. brasiliensis causou icterícia, hepatomegalia com acentuação no padrão lobular e coloração escura nos rins (BARROS et al., 1989). $\mathrm{Na}$ intoxicação natural por esta planta os ovinos apresentaram apatia, anorexia, emagrecimento progressivo, fotossensibilização, icterícia, incoordenação motora, andar compulsivo a esmo, decúbito e morte em casos mais severos (ILHA et al., 2001).

A literatura ainda relata a intoxicação natural por S. brasiliensis em búfalos; esses animais apresentaram apatia, perda de peso, diarreia e decúbito permanente (CORRÊA et al., 2008).

Quanto à intoxicação pelos APs, sabe-se que está relacionada com a susceptibilidade das diferentes espécies animais e com o balanço entre as reações de bioativação, desintoxicação e excreção desses alcaloides (CHEEKE, 1994). Os bovinos e equinos são bastante suscetíveis à intoxicação por Senecio, sendo 30 a 40 vezes mais sensíveis do que ovinos e caprinos (CRAIG et al., 1991), uma vez que, na maioria das vezes, estes últimos podem consumir a planta sem adoecerem (MÉNDEZ, 1993). Em particular, a resistência dos ovinos a ação hepatotóxica dos APs está relacionada às peculiaridades de sua flora ruminal (CRAIG et al., 1992) e aos sistemas enzimáticos do fígado, o que resulta em uma excelente detoxificação desses alcaloides (HUAN et al., 1998).

É importante destacar que o dano provocado pela ingestão de $S$. brasiliensis está relacionado com o tempo de exposição dos animais a esta planta, pois, em condições naturais, os animais passam longos períodos ingerindo pequenas quantidades da planta, caracterizando a exposição de forma crônica (TOKARNIA; DÖBEREINER, 2000). Na maioria dos casos os animais apresentam danos hepáticos sem a ocorrência de sinais clínicos, o que dificulta a realização do diagnóstico preciso. Uma alternativa para auxiliar o diagnóstico é a realização da biópsia hepática, embora seja necessário descartar intoxicações por outras plantas que 
causam cirrose hepática, intoxicações por nitrosaminas e aflatoxicose (TOKARNIA; DÖBEREINER, 2000; PILATI; BARROS, 2007).

\section{Sobre a toxicidade em seres humanos}

Em seres humanos há maior enfoque sobre a toxicidade causada pelos APs do que nas plantas que os possuem; por isso, aqui é dada maior ênfase nesses princípios tóxicos. Dessa forma, a principal manifestação da intoxicação por APs em seres humanos é a doença veno-oclusiva hepática (DOV), que é caracterizada por dor epigástrica com distensão abdominal devido à ascite (PRAKASH et al., 1999; MARIO, 2003). Na maioria dos casos a intoxicação ocorre diretamente através do uso medicinal crônico da planta na forma de chá (SPERL et al., 1995; CHEN; HUO, 2010). Recentemente, na Europa tem também chamado atenção problemas de saúde relacionados com hortaliças contaminadas com Senecio, como, por exemplo, rúcula vendida em supermercados contendo groundsel comum (Senecio vulgaris) (WIEDENFELD, 2011).

Sabe-se ainda, que a intoxicação pode ocorrer indiretamente através do consumo de produtos de origem animal contaminados por APs, como, por exemplo, leite (DICKINSON et al., 1976; MOLINEUX; JAMES, 1990; MOLYNEUX et al., 2011), ovos (EDGAR; SMITH, 2000) ou até mesmo através de mel (DEINZER et al., 1977; BOPPRÉ et al., 2005). Apesar dos estudos evidenciarem a passagem desses APs através do leite (DICKINSON et al., 1976; MOLINEUX; JAMES, 1990; HOOGENBOOM et al., 2011), até o momento não há descrições de intoxicações em seres humanos decorrente dessa via de exposição. Porém, vale ressaltar que Medeiros et al. (1999) mostraram que ratos expostos à ração contendo leite de cabra contaminado com um dos APs, monocrotalina, apresentaram alterações hepáticas e pneumotóxicas. Contudo, até o momento, cabe ressaltar que nenhum desses casos refere-se especificamente à $S$. brasiliensis.

Fica, pois, evidente, que o homem está exposto aos APs através de diferentes fontes, sendo a duração de exposição fator decisivo para causar toxicidade aguda, sub-aguda ou crônica. A intoxicação aguda é caracterizada por hemorragia, necrose, hepatomegalia e ascite; já a intoxicação subaguda é caracterizada pela reincidência de hepatomegalia e ascite, bem como proliferação endotelial e oclusão das veias hepáticas, culminando na toxicidade crônica com surgimento da DOV, podendo ser fatal em alguns casos (PRAKASH et al., 1999; FU et al., 2004). Estima-se que 20\% dos indivíduos intoxicados por APs cheguem ao óbito e que cerca de 50\% recuperam-se dentro de poucas semanas, sendo que dos indíviduos restantes, em torno de $20 \%$, parecem estar clinicamente recuperados, porém pode haver recidivas culminando no desenvolvimento da DOV e cirrose após alguns anos (PRAKASH et al., 1999).

Há evidências também da ação teratogênica de APs em seres humanos, uma vez que existem relatos de casos de DOV em recém-nascidos cujas mães consumiram, durante a gestação, chá de plantas que continham estes princípios tóxicos (HUXTABLE, 1985; ROULET et al., 1988; RASENACK et al., 2003).-

Dessa forma, constata-se que a toxicidade causada pelos APs caracteriza sério risco a saúde humana, através de consumo de alimentos contaminados e principalmente na forma de chá. Nesse sentido, umas das plantas com esses alcaloides mais estudada é Symphytum officinalis (confrei) que contém sete APs (RODE, 2002). Estudos demonstraram a presença desses compostos tóxicos em folhas frescas (MATTOCKS, 1980), raízes (BACH; THUNG; SCHAFFNER, 1989) e em preparações comerciais na forma de cápsulas e pílulas (RIDKER et al., 1985, BACH; THUNG; SCHAFFNER, 1989). Nos Estados Unidos e Canadá o uso interno do confrei foi banido após a ocorrência de intoxicações (RIDKER et al., 1985) e no Brasil em 1992; no entanto, produtos para aplicação tópica continuam liberados (PRAKASH et al., 1999).

Vale a pena ressaltar ainda, que mesmo com uso restrito, folhas de Heliotropium indicum e Eupatorium cannabinum, bem como o confrei são apontados como causadores de intoxicações em países desenvolvidos, como os Estados Unidos e Reino Unido (STICKEL; SEITZ, 2000; FU etal. , 2002; ROEDER; WIEDENFELD, 2009; MEI et al., 2010). 
No Brasil, S. brasiliensis é utilizada popularmente para diversas finalidades tais como: ferimentos da pele, vermífugo, dores estomacais e contra gripe (MAGNABOSCO et al., 1997; VISBISKI et al., 2003, TOMA, et al., 2004). No entanto, o uso indiscriminado desta planta pode causar danos à saúde humana, uma vez que foi relatado no nosso país casos de intoxicação em crianças e adultos que ingeriram chá de folhas de $S$. brasiliensis (MAGNABOSCO et al., 1997; TANIGUCHI et al., 2002). Até o presente momento, não há relatos de intoxicação em seres humanos devido à ingestão de alimentos contaminados com $S$. brasiliensis, porém é importante alertar a população quanto ao consumo dessa planta.

Dessa forma, fica evidente que a intoxicação por APs e, em particular, pela S. brasiliensis é uma preocupação em saúde pública, não se restringindo apenas em problema na agropecuária.

\section{Sobre medidas profiláticas das intoxicações por plantas}

As medidas profiláticas usadas para minimizar a incidência de intoxicação dos animais de produção, consistem principalmente em retirar a planta dos pastos; pode-se também empregar a técnica do controle biológico, através do uso, por exemplo, de insetos na pastagem, controlando a dispersão da planta no campo (ILHA et al., 2001; RIET-CORREA; MEDEIROS, 2001). Alguns autores sugerem a transferência de flora ruminal de ovinos para bovinos com objetivo de transferir a resistência a esses últimos (SOARES et al., 2000). No entanto, essas medidas profiláticas são pouco divulgadas e nem sempre apresentam uma boa eficácia contra a intoxicação de plantas tóxicas em animais. Uma alternativa para melhorar este quadro seria o desenvolvimento de centros de informações sobre enfermidades causadas pela ingestão de plantas tóxicas em animais domésticos, com o intuito de noticiar características das doenças e do comportamento fenológico das plantas tóxicas de maior relevância para a pecuária (KARAM et al., 2011).

\section{Considerações finais}

A intoxicação por $S$. brasiliensis, além de ser um problema que leva a perdas econômicas significativas para a pecuária no Brasil, é também uma preocupação em saúde pública, uma vez que pode afetar diretamente ou indiretamente a saúde dos seres humanos. Assim, fica evidente a importância do conhecimento e a divulgação sobre a profilaxia e o risco da exposição ao $S$. brasiliensis, bem como a realização de estudos para identificar a presença de princípios ativos tóxicos em outras plantas que apresentam danos para a saúde pública e veterinária.

\section{Agradecimento}

À Fundação de Amparo à Pesquisa do Estado de São Paulo (FAPESP).

\section{Referências}

BACH, N.; THUNG, S. N.; SCHAFFNER, F. Confrey herb teainduced hepatic veno-occlusive disease. The American Journal of Medicine, Tucson, v. 87, n. 1, p. 97-99, 1989.

BARROS, C. S. L.; CASTILHOS, L. M. L.; RISSI, D. R.; KOMMERS, G. D.; RECH, R. R. Biópsia hepática no diagnóstico da intoxicação por Senecio brasiliensis (Asteraceae) em bovinos. Pesquisa Veterinária Brasileira, Seropédica, v. 27, n. 1, p. 53-60, 2007.

BARROS, C. S. L.; CASTILHOS, L. M. L.; RISSI, D. R.; KOMMERS, G. D.; RECH, R. R.; METZDORF, L. L.; PEIXOTO, P. V. Ocorrência de surtos de intoxicação por Senecio spp. (Compositae) em bovinos no Rio Grande do Sul. Pesquisa Veterinária Brasileira, Seropédica, v. 7, n. 4, p. 101-107, 1987.

BARROS, C. S. L.; METZDORF, L. L.; SANTOS, M. N.; BARROS, S. S.; PEIXOTO, P. V. Intoxicação experimental por Senecio brasiliensis (Compositae) em ovinos. Pesquisa Veterinária Brasileira, Seropédica, v. 9, n. 3/4, p. 55-67, 1989.

BASILE, R. J.; DINIZ, J. M. F.; OKANO, W.; CIRIO, S. M.; LEITE, L. C. Intoxicação por Senecio spp. (Compositae) em bovinos no sul do Brasil. Acta Scientiae Veterinariae, Porto Alegre, v. 33, n. 1, p. 63-68, 2005.

BOPPRÉ, M.; COLEGATE, S. M.; EDGAR, J. A. Pyrrolizidine alkaloids Echium vulgare honey found in pure pollen. Journal of Agricultural and Food Chemistry, Washington, v. 53, n. 3, p. 594-600, 2005.

BRASIL - MINISTÉRIO DA SAÚDE. SECRETARIA NACIONAL DE VIGILÂNCIA SANITÁRIA. Portaria SNVS no 19, de 30.01.1992. Proibe o uso de confrei (Symphytum officinale L.) em preparações para uso interno. Brasília: Secretaria Nacional de Vigilância Sanitária, 1992. 
CABRERA, A. L.; KLEIN, R. M. Compostas 2, tribo Senecioneae. Itajaí: Flora Ilustrada Catarinense, 1975. 222 p.

CARVAlHO, G. S. T.; MAUGE, G. C. Ação tóxica de Senecio brasiliensis Lessing, Fam. Compositae. Revista da Faculdade de Medicina Veterinária e Zootecnia da Universidade de São Paulo, São Paulo, v. 3, n. 3, p. 131-136, 1946.

CHEEKE, P. R. Toxicity and metabolism of pyrrolizidine alkaloids. Journal of Animal Science, Champaign, v. 66, n. 9, p. 2343-2350, 1988.

CHEEKE, P. R. A review of the functional and evolutionary roles of the liver in the detoxication os poisonous plants, with special reference to pyrrolizidine alkaloids. Veterinary and Human Toxicology, Manhattan, v. 36, p. 240-247, 1994.

CHEEKE, P. R. Natural toxicants in feeds, foragens, and poisonous plants. Danville: Interstate Publishers, 1998. 479 p.

CHEN, Z.; HUO, J. R. Hepatic veno-occlusive disease associated with toxicity of pyrrolizidine alkaloids in herbal preparations. Netherlands Journal of Medicine, Alphen aan den Rijn, v. 68, n. 6, p. 252-260, 2010.

CONNOR, H. E. The poisonous plants in New Zealand. Wellington: E. C. Keating, Government Printer, 1977. 247 p.

CORRÊA, A. M. R.; BEZERRA, P. S. J.; PAVARINI, S. P.; SANTOS, A. S.; SONNE, L.; ZLOTOWSKI, P.; GOMES, G.; DRIEMEIER, D. Senecio brasiliensis (Asteraceae) poisoning in murrah buffaloes in Rio Gande do Sul. Pesquisa Veterinária Brasileira, Seropédica, v. 28, n. 3, p. 187-189, 2008.

CORRÊA, P. Dicionário das plantas do Brasil e das exóticas cultivadas. Rio de Janeiro: Instituto Brasileiro de Desenvolvimento Florestal, 1984. 4330 p.

CRAIG, A. M.; LATHAM, C. J.; BLYTHE, L. L.; SCHOMOTZER, W. B. Metabolism of toxic pyrrolizidine alkaloids from tansy ragwort (Senecio jacobaea) in ovine ruminal fluid under anaerobic conditions. Applied and Environmental Microbiology, Washington, v. 58, n. 9, p. 2730-2736, 1992.

CRAIG, A. M.; PEARSON, E. G.; MEYER, C.; SCHMITZ, J. A. Serum liver enzyme and histopathologic changes in calves with chronic and chronic-delayed Senecio jacobaea toxicosis. American Journal of Veterinary Research, Schaumburg, v. 52, n. 12, p. 1969-1978, 1991.

CULlEN, J. M. Doenças do fígado e do sistema biliar. In: MCGAVIN, M. D.; ZACHARY, J. F. (Ed.). Bases da patologia em veterinária. 4 ed. Rio de Janeiro: Elsevier, 2009. p. 418-455.

CURIAL, O.; GUIMARÃES, J. P. Cirrose hepática enzoótica no cavalo. Memórias do Instituto Oswaldo Cruz, Rio de Janeiro, v. 56, p. $635-643,1958$.

DALMOLIN, D. P.; RICCI, E. L.; TELLOLI, C. S.; SPINOSA, H. S.; GÓRNIAK, S. L. Efeitos da exposição ao Senecio brasiliensis durante a gestação no desenvolvimento físico e reflexológico da prole de ratas. In: SEMANA CIENTÍFICA BENJAMIN EURICO MALUCELLI, XIX, 2010, São Paulo. Anais... São Paulo: USP. v. 4, 2010. p. 112-113.

DEINZER, M. L.; THOMSON, P. A.; BURGETT, D. M.; ISAACSON, D. L. Pyrrolizidine alkaloids: their occurrence in honey from Tansy Ragwort (Senecio jacobaea L.). Science, Washington, v. 195, n. 4277, p. 497-499, 1977.

DICKINSON, J. O.; COOKE, M. P.; KING, R. R.; MOHAMED, P. A. Milk transfer of pyrrolizidine alkaloids in cattle. Journal of the
American Veterinary Medical Association, Schaumburg, v. 169, n. 11, p. 1192-1196, 1976.

EDGAR, J. A.; SMITH, L. W. Transfer of pyrrolizidine alkaloids into eggs: Food safety implications. In: Natural and select synthetic. Biological implications. ACS, Symposium Series American Chemical Society, Washington, v. 745, p. 118-128, 2000.

ELIAS, F.; LATORRE, A. O.; PÍPOLE, F.; HARAGUCHI, M.; GÓRNIAK, S. L.; HUEZA, I. M. Haematological and immunological effects of repeated dose exposure of rats to integerrimine N-oxide from Senecio brasiliensis. Food and Chemical Toxicology, Kidlington, v. 49, n. 1, p. 2313-2319, 2011.

FU, P. P.; XIA, Q.; LIN, G. Pyrrolizidine alkaloids - genotoxicity, metabolism enzymes, metabolic activation, and mechanism. Drug Metabolism Reviews, London, v. 36, n. 1, p. 1-55, 2004.

FU, P. P.; XIA, Q.; LIN, G.; CHOU, M. W. Genotoxic Pyrrolizidine Alkaloids - Mechanisms leading to DNA adduct formation and tumorigenicity. International Journal of Molecular Sciences, Basel, v. 3, n. 1, p. 948-964, 2002.

GAVA, A.; BARROS, S. L. C. Senecio spp. Poisoning of horses in south Brazil. Pesquisa Veterinária Brasileira, Seropédica, v. 17, n. 1, p. 36-40, 1997.

GRECCO, F. B.; SCHILD, A. L.; SOARES, M. P., MARCOLONGO-PEREIRA, C.; ESTIMA-SILVA, P.; SALLIS, E. S. V. Aspectos epidemiológicos e padrões de lesões hepáticas em 35 surtos de intoxicações por Senecio spp. em bovinos no sul do Rio Grande do Sul. Pesquisa Veterinária Brasileira, Seropédica, v. 30, n. 5, p. 389-397, 2010.

HENRIQUES, A. T.; LIMBERGER, R. P.; KERBER, V. A.; MORENO, P. R. H. Alcaloides: generalidades e aspectos básicos. In: SIMÕES, C. M. O.; SCHENKEL, E. P.; GOSMANN, G.; POLAZZO-MELLO, J. C.; MENTZ, L. A.; PETROVICK, P. R. (Ed.). Farmacognosia da planta ao medicamento. 5. ed. Florianópolis/Porto Alegre: Editora da UFSC/UFRGS, 2004. p. 765-791.

HIND, D. J. N. A Checklist of the Brazilian Senecioneae (Compositae). Kew Bulletin, Kew, v. 48, n. 2, p. 279-295, 1993.

HOOGENBOOM, L. A. P.; MULDER, P. P. J.; ZEILMAKER, M. J.; VAN DEN TOP, H. J.; REMMELINK, G. J.; BRANDON, E. F. A.; KLIJNSTRA, M.; MEIJER, G. A. L.; SCHOTHORST, R.; VAN EGMOND, H. P. Carry-over of pyrrolizidine alkaloids from feed to milk in dairy cows. Food Additives and Contaminants, York, v. 38, n. 3, p. 359-372, 2011.

HUAN, J. Y.; MIRANDA, C. L.; BUHLER, D. R.; CHEEKE, P. R. Species differences in the hepatic microsomal enzyme metabolism of the pyrrolizidine alkaloids. Toxicology Letters, Clare, v. 99, n. 2, p. 127-137, 1998.

HUXTABLE, R. Human embryotoxicity of pyrrolizidine-containing drugs. Hepatology, Baltimore, v. 9, n. 3, p. 510-511, 1985.

ILHA, M. R. S.; LORETTI, A. P.; BARROS, S. S.; BARROS, C. S. L. Intoxicação espontânea por Senecio brasiliensis (Asteraceae) em ovinos no Rio Grande do Sul. Pesquisa Veterinária Brasileira, Seropédica, v. 21, n. 3, p. 123-138, 2001.

JOHNSON, A. E.; MOLYNEUX, R. J.; MERRIL, G. B. Chemistry of toxic range plants. Variation in pyrrolizidine alkaloid content of Senecio, Amsinckia, and Crotalaria species. Journal of Agricultural and Food Chemistry, Washington, v. 33, n. 1, p. 50$55,1985$. 
KARAM, F. S. C.; MÉNDEZ, M. C.; JARENKOW, J. A.; RIETCORREA, F. Fenologia de quatro espécies tóxicas de Senecio (Asteraceae) na região Sul do Rio Grande do Sul. Pesquisa Veterinária Brasileira, Seropédica, v. 22, n. 1, p. 33-39, 2002.

KARAM, F. S. C.; MOTTA, A. Pyrrolizidine alkaloids poisoning in cattle in he state of Rio Grande do Sul, Brazil. In: RIET-CORREA, F.; PFISTER, J.; SCHILD, A. L.; WIERENGA, T. (Ed.). Poisoning by plants, mycotoxins and related toxins. Wallingford: $\mathrm{CAB}$ International, 2011. p. 175-178.

KARAM, F. S. C.; SCHILD, A. L.; MELLO, J. R. B. Intoxicação por Senecio spp. em bovinos no Rio Grande do Sul: condições ambientais favoráveis e medidas de controle. Pesquisa Veterinária Brasileira, Seropédica, v. 31, n. 7, p. 603-609, 2011.

KARAM, F. S. C.; SOARES, M. P.; HARAGUCHI, M.; RIETCORREA, F.; MÉNDEZ, M. C.; JARENKOW, J. A. Aspectos epidemiológicos da seneciose na região sul do Rio Grande do Sul. Pesquisa Veterinária Brasileira, Seropédica, v. 24, n. 4, p. 191198, 2004.

LORENZI, H. Manual de identificação e controle de plantas daninhas. 4. ed. Nova Odesa. Editora: Plantarum, 1994. p. 86-87.

LUCENA, R. B.; PIEREZAN, F.; KOMMERS, G. D.; IRIGOYEN, L. F.; FIGHERA R. A.; BARROS, C. S. L. Doenças de bovinos no Sul do Brasil: 6.706 casos. Pesquisa Veterinária Brasileira, Seropédica, v. 30, n. 5, p. 428-434, 2010.

MACEL, M.; VRIELING, K.; KLINKHAMER, P. G. L. Variation in pyrrolizidine alkaloid patterns of Senecio jacobaea. Phytochemistry, Oxford, v. 65, n. 7, p. 865-873, 2004.

MACLACHLAN, N. J.; CULLEN, J. M. Fígado, sistema biliar e pâncreas exócrino. In: CARLTON, E M.; MCGAVIN, D. E. (Ed.). Patologia veterinária especial de Thomson. 2. ed. Porto Alegre: Artmed, 1998. p. 95-131.

MAGNABOSCO, E. M.; RIVERA M. L.; PROLLA I. R.; DE VERNEY Y. M.; DE MELLO E. S. Hepatic veno-oclusive disease: report of case, Journal of Pediatrics, Nova York, v. 73, n. 2, p. 115-118, 1997.

MARIO, C. Hepatic sinusoidal-obstruction syndrome: toxicity of pyrrolizidine alkaloids. Journal of Hepatology, Amsterdam, v. 39, n. 3, p. 437-446, 2003.

MATTOCKS, A. R. Toxicity of Pyrrolizidine Alkaloids. Nature, London, v. 217, n. 1, p. 723-728, 1968.

MATTOCKS, A. R. Toxic pyrrolizidine alkaloids in comfrey, Lancet, New York, v. 29, n. 1, p. 1136-1137, 1980.

MATTOCKS, A.; DRIVER, H. E.; BARBOUR, R. H.; ROBINS, D. J. Metabolism and toxicity of synthetic analogues of macrocyclic diester pyrrolizidine alkaloids. Chemico-Biological Interactions, Clare, v. 58, n. 1, p. 95-108, 1986.

MEDEIROS, R. M. T.; GÓRNIAK, S. L.; GUERRA, J. L. Effects of milk from goat fed Crotalaria spectabilis seeds on growing rats. Brazilian Journal of Veterinary Research and Animal Science, São Paulo, v. 36, n. 2, p. 12-19, 1999.

MEI, N.; GUO, L.; PETER, P.; FU, P. O.; FUSCOE, J. C.; YANG LUAN, Y.; TAO CHEN, T. Metabolism, genotoxicity and carcinogenicity of comfrei. Journal of Toxicology and Environmental Health B, Ottawa, v. 13, n. 7-8, p. 509-526, 2010. MÉNDEZ, M. C. Intoxicação por Senecio spp. In: RIET-CORREA, F.; MÉNDEZ, M. C.; SCHILD, A. L. (Ed.). Intoxicações por plantas e micotoxicoses em animais domésticos. Pelotas: Editora Hemisfério Sul do Brasil, 1993. p. 43-57.

MÉNDEZ M., C.; RIET-CORREA, F. Plantas tóxicas e micotoxicoses. Pelotas: Editora e Gráfica Universitária, 2008. 298 p.

MÉNDEZ, M. C.; RIET-CORREA, F.; SHILD, A. L.; WALTER, M. Intoxicação experimental por cinco espécies de Senecio em bovinos e aves. Pesquisa Veterinária Brasileira, Seropédica, v. 10, n. 3/4, p. 63-69, 1990.

MOLYNEUX, R. J.; GARDNER, D. L.; COLEGATE, S. M.; EDGAR, J.A. Pyrrolizidine alkaloid toxicity in livestock: a paradigm for human poisoning? Food Additives and Contaminants, York, v. 28, n. 3, p. 293-307, 2011.

MOLINEUX, R. J.; JAMES, L. F. Pyrrolidizine alkaloids in milk: thresholds of intoxication. Veterinary and Human Toxicology, Manhattan, v. 32, n. 1, p. 94-103, 1990.

MORAES, E. C. F. Contribuição ao estudo químico-toxicológico do Senecio brasiliensis Less. 1952. 90 f. Tese (Livre Docência) Universidade de São Paulo, São Paulo. 1952.

MOTIDOME, M.; FERREIRA, P. C. Alcaloides do genus Senecio. Revista da Faculdade de Farmácia Bioquímica da Universidade de São Paulo, São Paulo, v. 4, p. 13-44, 1966.

PILATI, C.; BARROS, C. S. L. Intoxicação experimental por Senecio brasiliensis (Asteracea) em equinos. Pesquisa Veterinária Brasileira, Seropédica, v. 27, n. 7, p. 287-296, 2007.

PRAKASH, A. S.; PEREIRA, T. N.; REILLY, P. E. B.; SEAWRIGHT, A. A. Pyrrolizidine alkaloids in human diet. Mutation Research/Genetic Toxicology and Environmental Mutagenesis, Amsterdam, v. 443, n. 1-2, p. 53-67, 1999.

RADOSTITS, O. M.; GAY, C. C.; BLOOD, D. C.; HINCHLIFF, K. W.; ARUNDEL, J. H.; FIGUEIREDO, C.; JACOBS, D. E.; LESLIE, B. O.; MCKENZIE, R. A.; BILDFELL, R. J. Clínica veterinária: um tratado de doenças de bovinos, ovinos, suínos, caprinos e equinos. Rio de Janeiro: Guanabara, 2002. 1737 p.

RASENACK, R.; MULLER, C.; KLEINSCHMIDT, M.; RASENACK, J.; WIEDENFELD, H. Veno-occlusive disease in a foetus caused by pyrrolizidine alkaloids of food origin. Fetal Diagnosis and Therapy, Basel, v. 18, n. 4, p. 223-225, 2003.

RIDKER, P. M.; SEITARO, O.; MCDERMOTT, W. V.; TREY, C.; HUXTABLE, R. J. H. Hepatic venocclusive disease associated with the consumption of pyrrolizidine-containing dietary supplements. Gastroenterology, Philadelphia, v. 88, n. 4, p. 1050-1054, 1985.

RIET-CORREA, F.; MEDEIROS, M. R. T. Intoxicações por plantas em ruminantes no Brasil e no Uruguai: importância econômica, controle e riscos para a saúde pública. Pesquisa Veterinária Brasileira, Seropédica, v. 21, n. 1, p. 38-42, 2001.

RIET-CORREA, F.; MÉNDEZ, M. C.; SCHILD A. L. Intoxicações por plantas e micotoxicoses em animais domésticos. Pelotas: Editorial Hemisfério Sul do Brasil, 1993. 340 p.

RIET-CORREA, F.; SCHILD, A. L.; MÉNDEZ, M. C.; OLIVEIRA, J. A.; GIL-TURNES, C.; GONÇALVES, A. Relatório de atividades e doenças da área de influência no período de 1978/1982. Pelotas: Laboratório Regional de Diagnóstico, 1983. p. 42-44.

RIET-CORREA, F.; SOARES, M. P.; MENDEZ, M. R. T. Intoxicações em eqüinos no Brasil. Ciência Rural, Santa Maria, v. 28, n. 4, p. 715-722, 1998. 
RISSI, D. R.; RECH, R. R.; PIEREZAN, F.; GABRIEL, A. L.; TROST, M. E.; BRUM, J. S.; KOMMERS, G. D.; BARROS, C. S. L. Intoxicações por plantas e micotoxinas associadas a plantas em bovinos no Rio Grande do Sul: 461 casos. Pesquisa Veterinária Brasileira, Seropédica, n. 27, p. 261-268, 2007.

RODE, D. Comfrey toxicity revisited. TRENDS in Pharmacological Sciences, London, v. 23, n. 11, p. 497-499, 2002.

ROEDER, E.; WIEDENFELD, H. Pyrrolizidine alkaloids in medicinal plants of Mongolia, Nepal and Tibet. Pharmazie, Eschborn, v. 64, n. 1, p. 699-716, 2009.

ROULET, M.; LAURINI, R.; RIVIER, L.; CALAME, A. Hepatic veno-occlusive disease in newborn infant of a woman drinking herbal tea. Journal of Pediatrics, Nova York, v. 112, n. 3, p. 433$436,1988$.

SANDINI, T. M.; UDO, M. S. B.; SPINOSA, H. S.; BERNARDI, M. M. Exposure to Senecio brasiliensis during the organogenesis period impaired the physical and reflexologic development of rat offspring. In: CONGRESS OF THE EUROPEAN SOCIETIES OF TOXICOLOGY, 47, 2011, Paris. Proceedings... Paris: EUROTOX, 2011. p. 2238.

SANTOS, J. C. A.; RIET-CORREA, F.; SIMÕES, S. V. D.; BARROS, C. S. L. Patogênese, sinais clínicos e patologia das doenças causadas por plantas hepatotóxicas em ruminantes e eqüinos no Brasil. Pesquisa Veterinária Brasileira, Seropédica, v. 28, n. 1, p. 1-14, 2008.

SHIRATORI, Y.; TANANKA, M.; KAWASE, T.; SHIINA. S.; KOMATSU, Y.; OMATA, M. Quantification of sinusoidal cell function in vivo. Seminars in Liver Disease, Nova York, v. 13, n. 1, p. 39-49, 1993.

SOARES, M. P.; RIET-CORREA, F.; MÉNDEZ, M. C.; ROSA, F. G.; CARREIRA, E. G. Controle biológico de Senecio spp. com pastoreio de ovinos, Corrientes, Argentina, 2000. In: REUNIÓN ARGENTINA DE PATOLOGIA VETERINÁRIA, II, 2000, Corrientes. Anais... Corrientes: Facultad de Ciências Veterinárias, Universidad Nacional Del Nordeste, 2000. Versão eletrônica.

SOUZA, F. O. Asteraceae no Parque Estadual da Ilha do Cardoso, Cananéia, SP. 2007. 159 f. Dissertação (Mestrado em Botânica) - Instituto de Botânica da Secretaria de Estado do Meio Ambiente, São Paulo. 2007.

SPERL, W.; STUPPNER, H.; GASSNER I.; JUDMAIER, W.; DIETZE, O.; VOGEL, W. Reversible hepatic veno-occlusive disease in an infant after consumption of pyrrolizidine-containing herbal tea. European Journal of Pediatrics, Nova York, v. 154, n. 2, p. 112-116, 1995.
SPINOSA, H. S.; GÓRNIAK, S. L.; PALERMO-NETO, J. Toxicologia aplicada à Medicina Veterinária. São Paulo: Editora Manole, 2008. 942 p.

STEGELMEIER B. L. Pyrrolizidine alkaloid-containing toxic plants (Senecio, Crotalaria, Cynoglossum, Amsinckia, Heliotropium, and Echium spp.). Veterinary Clinics of North America: Food Animal Practice, Philadelphia, v. 27, n. 2, p. 419 428,2011

STICKEL, F.; SEITZ, H. K. The efficacy and safety of comfrey. Public Health Nutrition, Cambridge, v. 3, n. 4A, p. 501-508, 2000.

TANIGUCHI, A. N. R.; CÉLIA, L.; VIEIRA, S. M.; FERREIRA, C. T.; ZAFFONATTO, D.; CERSKI, T.; SILVEIRA, T. R. Relato de caso - Doença veno-oclusiva induzida por chá de Senecio brasiliensis. Revista do Hospital de Clínicas de Porto Alegre e Faculdade de Medicina da Universidade Federal do Rio Grande do Sul, Porto Alegre, v. 22, n. 1, p. 183-184, 2002.

TOKARNIA, C. H.; DÖBEREINER, J. Plantas tóxicas do Brasil. Rio de Janeiro: Helianthus, 2000. 320 p.

TOMA, W.; TRIGO, J. R.; DE PAULA, A. C. B.; BRITO, A. R. M. S. Preventive activity of pyrrolizidine alkaloids from Senecio brasiliensis (Asteraceae) on gastric and duodenal induced ulcer on mice and rats. Journal of Ethonopharmacology, Leiden, v. 95, n. 2-3, p. 345-341, 2004

TORRES, M. B. A. M.; COELHO, K. I. R. Experimental poisoning by Senecio brasiliensis in calves: quantitative and semi-quantitative study on changes in the hepatic extracelular matrix and sinusoidal cells. Pesquisa Veterinária Brasileira, Seropédica, v. 28, n. 1, p. 43-50, 2008

TRIGO, R. J.; LEAL, I. R.; MATZENBACHER, N. I.; LEWINSOHN, T. M. Chemotaxonomic value of pyrrolizidine alkaloids in southern Brazil Senecio (Senecioneae: Asteraceae). Biochemical Systematic and Ecology, Oxford, v. 31, n. 1, p. 1011 1022, 2003.

VISBISKI, V. N.; WEIRICH NETO, P. H.; SANTOS, A. L. O uso popular da plantas medicinais no assentamento Guanabara, ImbaúPR. Publicatio UEPG: Ciências Exatas e da Terra, Ciências Agrária e Engenharia, Ponta Grossa, v. 9, n. 1, p. 13-20, 2003.

WIEDENFELD, H. Plants containing pyrrolizidine alkaloids: toxicity and problems. Food Additives and Contaminants, York, v. 28, n. 3 , p. $282-292,2011$. 